\title{
Collaborative Filtering Movie Recommendation System
}

\author{
Manjeet Singh $^{1}$ | Namita Goyal ${ }^{1}$
}

${ }^{1}$ IT (Information Technology), Maharaja Agrasen Institute of Technology,

To Cite this Article

Manjeet Singh and Namita Goyal, "Collaborative Filtering Movie Recommendation System", International Journal for Modern Trends in Science and Technology, 6(12): 471-473, 2020.

\section{Article Info}

Received on 16-November-2020, Revised on 09-December-2020, Accepted on 12-December-2020, Published on 21-December-2020.

\section{ABSTRACT}

Recommendation System plays an important role in today's era of e-commerce. From OTT platforms to the shopping application and music application everywhere we see that after watching a movie or buying an item, listening a song we are recommended with some other movie or item or song. Most of the time we select our next movie, item or song from the recommended one. In this paper I will give you a brief description of collaborative and user-based filtering. The data used in this research is taken from Movie Lens. The result obtained contains some movie recommendations.

KEYWORDS: Collaborative Filtering Method, Recommendation System, collaborative filtering, movie recommendation.

\section{INTRODUCTION}

Recommendation System works on the ratings, opinion of the person of different age groups, different genders. It helps in finding the user the content of his choice easily on the basis of the users which are similar to him/her. Every platform whether it is product-based company e.g. amazon, flipkart, social media e.g. Facebook, Twitter or any OTT platform e.g. amazon prime, Netflix all uses one or other kind of recommendation system to suggest item, posts or movies, web-series to their users.

Recommendation system help the users to find the things easily instead of letting them browse for hours to find the thing that they are looking for. It takes the data from the previous behavior of the user and also from the user of same type. After that different algorithms are used to forecast the items that they may be interested in.
Two main approaches to build a recommendation system are content based recommendation system in which the content that the user has purchased and liked. The other method is the collaborative filtering recommendation system on which we group users of the same kind and then based on their history we recommend the product to the user.

Many types of recommendation are available in the industry but still the research is going on to build the recommendation system which suggest the more accurate item to the user with less browsing and to give user a better experience.

\section{IDENTIFY, RESEARCH AND COLLECT IDEA}

Many algorithms have been made for recommendation system. Many techniques have been studied in the past decades. Some of the examples are ALS algorithm, recommendation 
system based on weighting technique, content based collaborative filtering. These techniques use the prior data and rating of the users. These systems are not that much accurate and research is still going on to improve the techniques.

\section{Implementation OF Collaborative Filtering USING KNN-ALGORITHM}

Nikita Sharma has represented the recommendation system. In this recommendation system the data set is taken from Movie Lens. In this recommendation system the ratings and the similarity between the users is used to recommend the movies. The movies are divided in to two sets: the rated and unrated movie sets. Then using the KNN algorithm movies are recommended to the user.In KNN, a data point is classified by a majority vote of its neighbors, with the data point being assigned to the class most common amongst its k-nearest neighbors, as measured by a distance function (these can be of any kind depending upon your data being continuous or categorical). If $\mathrm{k}=1$, then the data point is simply assigned to the class of its nearest neighbor-i.e., itself. In KNN (k nearest neighbors) the movies according to the users rating which are closer to the movies on which basis the recommendation is to be given are recommended. Below figure give the best understanding of the recommendation system using the knn algorithm.

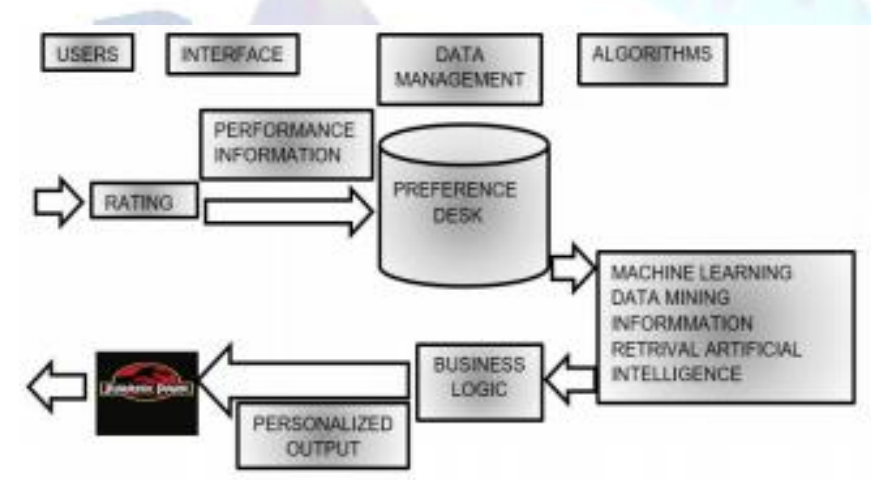

Fig. The workflow of the recommendation system.

The following figure will give you a brief description between content based and collaborative filtering.

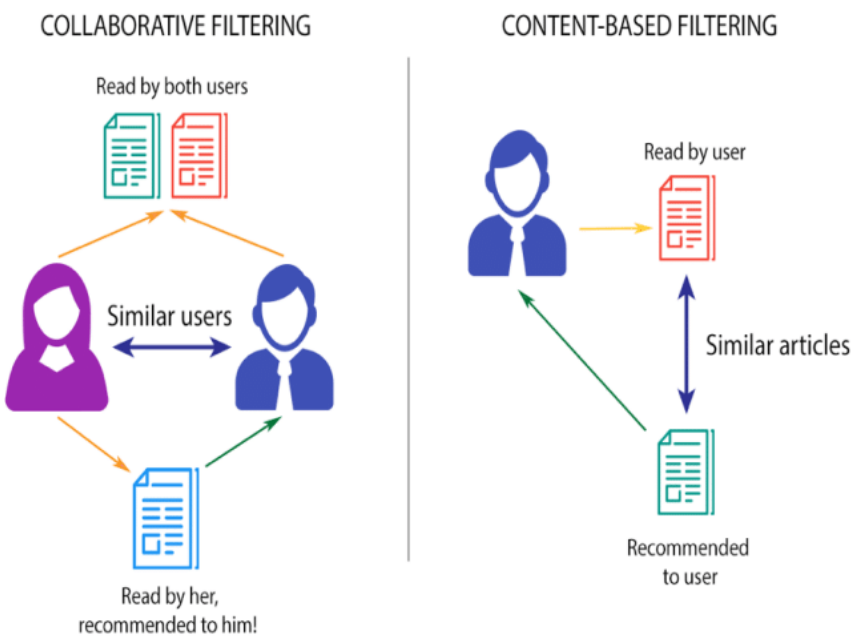

\section{A. Content Based Recommendation System}

In this type of filtering the user is recommended with the same type of items that the user see. In case of movie recommendation system only one type of the movie is suggested to the user. It can be on the basis of the genre or the casting of the movie. The content in this system usually uses keywords and in formativeness of a keyword is measured by TFIDF weight.

Limitations of the content-based recommendation are that the user is restricted to only a limited content. It is over specialization. One of the limitations is that to a new user this system is not able to recommend any thing.

\section{B. Limitation of the Collaborative Filtering}

Following are the limitations of the collaborative filtering:

1. When a new item is there in the data because of its low ratings it is being recommended to the user. So, this recommendation system creates the limitations to the new content and restrict its expansion.

2. With the increase in the new users and the new items in the data set the load on the system also increases. It reduces the accuracy of the system. Large data will take long time to process and this will also affect the speed of the system.

3. Some of the users do not give ratings to movies and this creates lot of difficulty in finding the nearest neighbor and give user a good recommendation.

4. Datasets used in processing contain various attributes of a user in form of information. There is risk of leakage of some confidential information present or this information can be modified to use in an inappropriate way. 


\section{CONCLUSION}

Several techniques are proposed for recommender systems are available today based on content based and, collaborative technique. Collaborative filtering technique is the most widely used technique and is able to handle scalability issue. It provides better recommendation for the users. In this paper it covers collaborative filtering approach on film trust movie data set to provide recommendation of movies for a particular user.

\section{REFERENCES}

1. Bobadilla J, Ortega F, Hernando A, Gutiérrez A (2013) Recommender systems survey. Knowle Based Syst 46:109-132. https://doi.org/10.1016/j.knosys.2013.03.012

2. Cui, Bei-Bei. (2017). Design and Implementation of Movie Recommendation System Based on Knn Collaborative Filtering Algorithm. ITM Web of Conferences. 12. 04008. 10.1051/itmconf/20171204008.

3. H. Zarzour, F. M. Mohamed, S. Chaouki, and C. Chemam, "An improved collaborative filtering recommendation algorithm for big data," Computational Intelligence and Its Applications, pp. 660-668, 2018.

4. https://www.hindawi.com/journals/misy/2019/3560968/ 5. J. Aranda, I. Givoni, J. Handcock, and D. Tarlow, An Online Social Network-Based Recommendation System, University of Toronto, Ontario, Canada, 2007.

6.http://www.ijfrcsce.org/download/browse/Volume_4/Dece mber_18_Volume_4_Issue_12/1546423733_31-12-2018

7.https://link.springer.com/chapter/10.1007\%2F978-981-131274-8_22

8.https:// heartbeat.fritz.ai/recommender-systems-with-pytho $\mathrm{n}$-part-ii-collaborative-filtering-k-nearest-neighbors-algorithmc8dcd5fd89b2

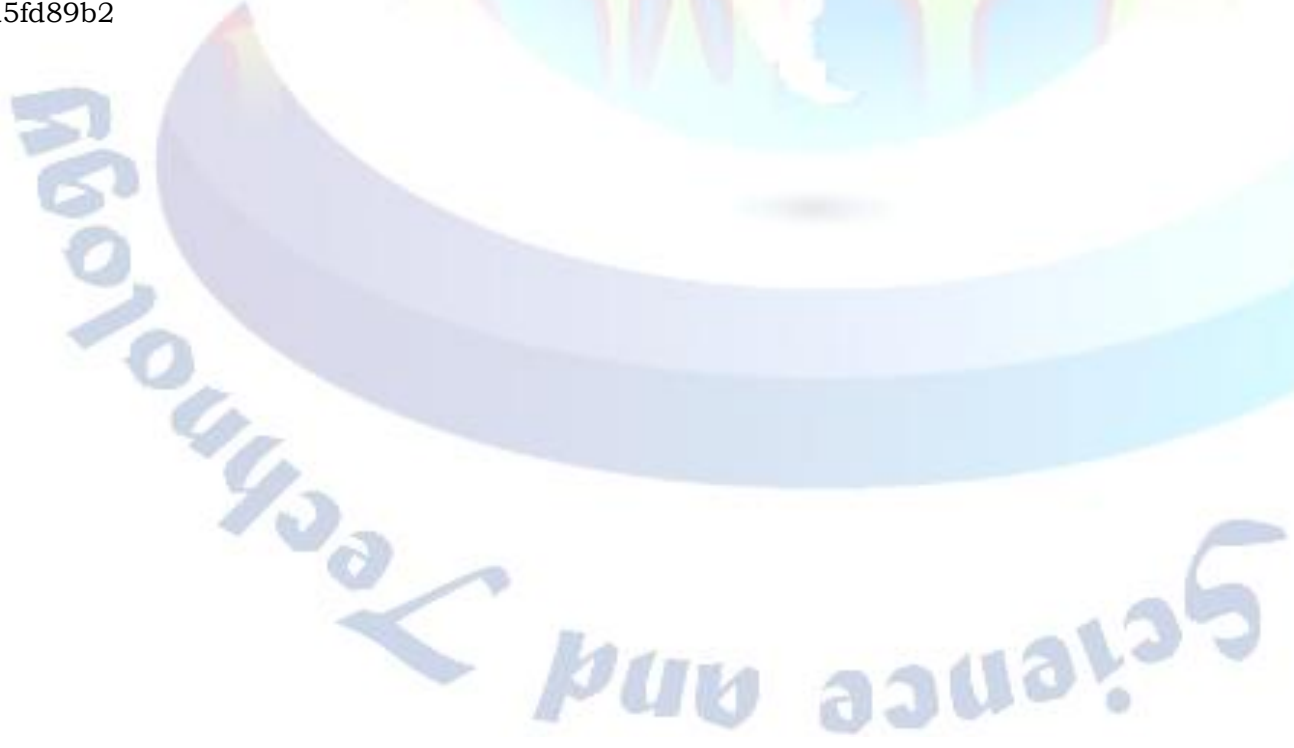

